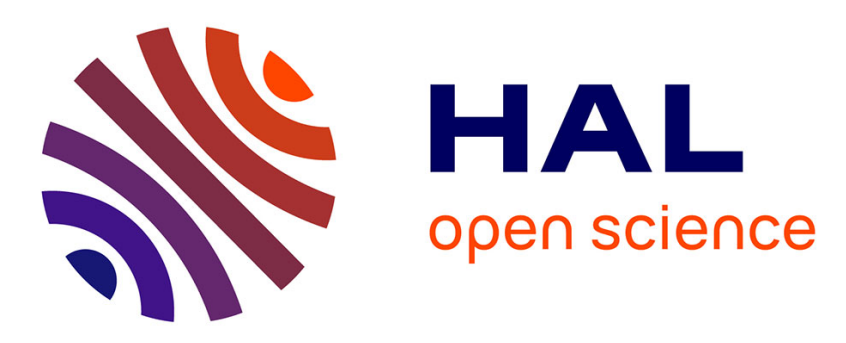

\title{
An Architectural Model for System of Information Systems
}

Majd Saleh, Marie-Helene Abel, Alok Mishra

\section{To cite this version:}

Majd Saleh, Marie-Helene Abel, Alok Mishra. An Architectural Model for System of Information Systems. ISDE15 Sixth International Workshop on Information Systems in Distributed Environment, Oct 2015, Rhodes, Greece. pp.411-420, 10.1007/978-3-319-26138-6_44 . hal-01282797

\section{HAL Id: hal-01282797 https://hal.science/hal-01282797}

Submitted on 7 Mar 2016

HAL is a multi-disciplinary open access archive for the deposit and dissemination of scientific research documents, whether they are published or not. The documents may come from teaching and research institutions in France or abroad, or from public or private research centers.
L'archive ouverte pluridisciplinaire HAL, est destinée au dépôt et à la diffusion de documents scientifiques de niveau recherche, publiés ou non, émanant des établissements d'enseignement et de recherche français ou étrangers, des laboratoires publics ou privés. 


\title{
An Architectural Model for System of Information Systems
}

\author{
Saleh Majd, Abel Marie-Hélène, and Mishra Alok \\ Sorbonne Universités, Université de Technologie de Compiègne, CNRS, UMR 7253 \\ Heudiasyc, Compiègne, France \\ \{majd.saleh, marie-helene.abel, alok.mishra\}@hds.utc.fr
}

\begin{abstract}
One of the most important aspects when designing and constructing an Information System is its architecture. This also applies to complex systems such as System of Information Systems (SoIS). Thus, we aim to propose an architectural model of System of Information Systems (SoIS). Though Architecture-based approaches have been promoted as a means of controlling the complexity of systems construction and evolution, what we really look for in this paper is an architectural model to aggregate services from already constructed systems. Nevertheless, it would be a good practice to compare the presented architecture of SoIS to other architecture-based approaches such as Service Oriented Architecture (SOA). Also, it is beneficial to examine how we can use the well-established standards of SOA for the designing of SoIS. In this paper we present an architectural model for System of Information Systems, and highlight the standards of Service Oriented Architecture that might help us in this task.
\end{abstract}

Keywords: System of Systems, System of Information Systems, Service Oriented Architecture, Architectural Model

\section{Introduction}

In recent years the work environment is becoming more competitive, as changes are taking place much faster than before. One of these changes happens with the services provided by Information Systems employed by companies, as these systems need to learn how to work and communicate with each other in order to aggregate services and produce new ones emerged from existing systems. Therefore, securing a competitive advantage does no longer rely only on efficiency, quality, and customer responsiveness. While each of these factors is important, the ability to deploy available solution to emergent problems by aggregating services of existing Information Systems and provide ease of access to these systems is of great importance. This makes innovation, flexibility, coordination, integration, and speed the new success factors of todays work environment. Those factors can be achieved through the concept of System of Information Systems (SoIS). While successful SoIS production provides the basis of great potentials, many development activities result in failure. The issue is that while 
many individual Information Systems work well as an independent system, they fail when incorporated as a component of a SoIS. The desired SoIS needs to connect Information Systems that cross organizational boundaries, come from multiple domains, and generates an overwhelming amount of information. Users struggle to deal with the information produced by each Information System independently by traversing through these systems and keeping track of the generated information separately. A solution might be found in a well-established architectural model of System of Information Systems that provides guidance to produce such solution. The SoIS operates as a single entry point for several Information Systems granting the user access to information produced from multiple Information Systems, and providing the ability to aggregate available services to even create an added value not possible to maintain when those systems were operating separately. Furthermore, this encompassing solution should also work in a distributed environment over a network as the Information Systems composing the SoIS could be geographically distributed. Distributed system development is becoming crucial due to the requirement of applications involving different devices and different sources of information. Management of distributed software development has more challenges and difficulties than conventional development [Mishra and Mishra, 2009] [Mishra and Alok, 2011]. The construction of SoIS, however, requires long-term projects that involve comprehensive organizational changes in terms of new approaches to system usage and different IT governance mechanisms, as well as changes in the roles and responsibilities of employees and Information Systems users in particular. To address this issue we believe that Service Oriented Architecture (SOA) should be utilized as a business transformation tool for solving the needs of SoIS.

This paper is organized as follows: section 2 will provide the definition of the notion of System of Systems (SoS). After that, in section 3, the SoIS architectural model is presented and then discussed by defining the concept of SoIS, and presenting an example following our architectural model of SoIS. Then, a comparison between SoIS and SOA in terms of similarities, differences, and limitations is presented in section 4 . Section 5 will present a discussion of the findings of this paper. Finally, we conclude with section 6 .

\section{Definition of System of Systems}

The notion of System of Systems (SoS) can be viewed as an evolution of the standard notion of systems. Many definitions of a SoS exist. An aggregate of systems leads to the creation of new forms of systems which may be either described within the framework of composite systems, or demonstrate additional features which add complexity to the description and may be referred to as System of Systems.

We can summarize the definitions mentioned in [Jamshidi, 2011] [Carlock and Fenton, 2001] [Manthorpe, 1996] [Rechtin and Maier, 2000]. In the 
light of the definitions mentioned in the literature, we can describe the notion of System of Systems (SoS) as the following; Systems of systems are large-scale integrated systems which are heterogeneous and independently operable on their own, but are networked together for a common goal. The goal, as mentioned before, may be cost, performance, robustness, etc. In other terms, A System of Systems is a super system comprised of other elements which themselves are independent complex operational systems and interact among themselves to achieve a common goal. Each element of a SoS achieves well-substantiated goals even if they are detached from the rest of the SoS.

\section{System of Information Systems}

In this section we propose a new system that combines the services from different Information Systems. First, we are going to define our new system as a System of Information Systems (SoIS). Then, we are going to present a generic architecture of the SoIS. Finally we propose an intial design for the SoIS that can serve as an example of the architecture

\subsection{Definition}

The notion of System of Information Systems is defined by [Carlsson and Stankiewicz, 1991] as "networks of agents interacting in a specific technology area under a particular institutional infrastructure for the purpose of creating, diffusing, and utilizing technology focused on knowledge, information, and competence flow." [Breschi and Malerba, 1996] describe SoIS as "the specific clusters of the firms, technologies, and industries involved in the generation and diffusion of new technologies and in the knowledge flow that takes place among them."

Based on the definitions provided we can summaries the features of SoIS as follows:

- SoIS addresses the impact of the interrelationships between different IS.

- SoIS is concerned with the flow of information and knowledge among different information systems.

- SoIS is responsible for generating information from the emergent IS.

- Information interoperability is a key issue when designing a SoIS.

\subsection{Architecture}

In this part we are going to present the generic architecture of the SoIS. As seen in (Fig. 1), the SoIS will aggregate services from several Information Systems (System A, System B etc.). These systems are working separately. Each of which has its own services and databases. The services of these systems are denoted as solid rectangles inside the system (Service 1A, Service 1B etc.). Information can be represented in different ways within different systems, thus, the SoIS 


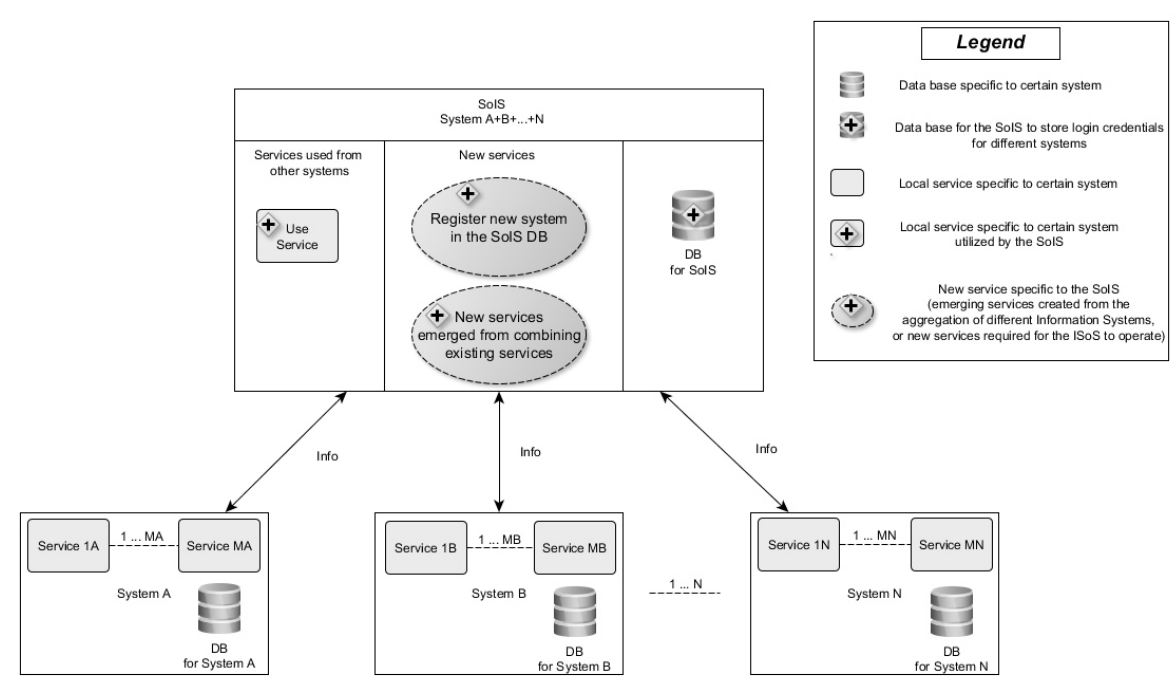

Fig. 1. Architectural model of the SoIS.

might have trouble access information. Therefore, there is a growing need for a solution to this interoperability issue. A solution to the problem of assuring interoperability within the SoIS is to control the communication medium among the systems. Two methods, outlined by [Bowen and Sahin, 2010], include:

- Creating a software model of each system, where the software model collects data from the system and generates the outputs.

- Creating a common language to describe data, where each system can represent its data such that other systems may interpret.

Due to overhead restrictions on architecture and a required common language, it is not often that individual software models are created for the various systems. Therefore, the most widely used approach to ensure interoperability within a SoIS is to standardize the language of data interpretation throughout the SoIS. The SoIS is represented as a group of services and a database. The services residing in the SoIS can either be utilization of existed services from the Information Systems comprising the SoIS, or emerging services created from the aggregation of different Information Systems. Existing services re denoted with hard lines while emergent services are denoted with dotted lines. In addition, the two headed arrows linking the SoIS with the Information systems represent information path between the SoIS and the different Information Systems.

\subsection{Example}

As mentioned earlier, the SoIS is composed of several Information Systems. In this part we will take two Information Systems to create a SoIS from them 
by following the architecture presented earlier in (Fig. 1). These Information Systems are TiddlyWiki [tid, 2015] and MEMORAe. First we will take a look at each of these systems separately and highlight their functionality as independent systems, before unveiling their role as parts of the SoIS.

TiddlyWiki: Technically, a TiddlyWiki is just an HTML page with a rather large JavaScript section that takes care of displaying all the contents, and provides the interactive tools for their manipulation. The actual text of the page is not immediately visible. It is stored in a set of invisible DIV elements, called tiddlers. The content of the DIV tags is wiki text, i.e. text with a simple markup language, similar to (old) emails. When the user clicks on a tiddler name to show its content, the JavaScript rendering machine translates the wiki text into HTML. The text may also contain macro fragments that trigger the actions of subroutines. Other tiddlers are interpreted as a stylesheet or a JavaScript plugin. When the user asks for a tiddler to be edited, this is replaced inline by a form, and the user is presented with the original text [Bagnoli et al., 2006].

MEMORAe: As defined by [Ala Atrash, 2014], MEMORAe approach is to manage heterogeneous information resources within organizations. The approach is comprised of a semantic model (called MEMORAe-core 2) and a web platform (called MEMORAe) which is based on the semantic model. The model and the platform make together a support to enhance the process of organizational learning. The MEMORAe project uses the Semantic Web standards, therefore, the ontologies occurring in the system are written in OWL. Users registered in the MEMORAe system can access one or more knowledge bases. When a base is chosen, a user can view a semantic map of concepts related to the selected base. Then, a user can create and share resources around the concepts of the map.

After being introduced to the components of the SoIS, the services of both those systems are present in Table 1 . Table 2 shows the services available in the SoIS either employed from one of the Information Systems or emerged from the existing services.

Table 1. Available services in the Information Systems comprising the SoIS

\begin{tabular}{|c|l|l|}
\cline { 2 - 3 } \multicolumn{1}{c|}{} & \multicolumn{1}{c|}{$\begin{array}{c}\text { TiddlyWiki } \\
\text { (System A) }\end{array}$} & \multicolumn{1}{c|}{$\begin{array}{c}\text { MEMORAe } \\
\text { (System B) }\end{array}$} \\
\hline \multirow{4}{*}{ Available Service } & Create Wiki pages & Index resources \\
\cline { 2 - 3 } & Edit Wiki pages & Annotate resources as a whole \\
\cline { 2 - 3 } & Use macro fragments in Wiki pages & Annotate resources as parts \\
\cline { 2 - 3 } & Share resources \\
\hline
\end{tabular}

The idea behind this example is to be able to use MEMORAe system potential in indexing and sharing resources alongside annotating them for the benefit 
Table 2. Available services in the SoIS

\begin{tabular}{|l|l|}
\cline { 2 - 2 } \multicolumn{1}{c|}{} & \multicolumn{1}{c|}{ SoIS } \\
\hline \multirow{4}{*}{ Availablem $\mathbf{A}+$ System B $)$} \\
\hline & Create Wiki pages \\
\hline & Edit Wiki pages \\
\cline { 2 - 2 } & Use macro fragments in Wiki pages \\
\cline { 2 - 2 } & Index Wiki Pages \\
\cline { 2 - 2 } & Share Wiki Pages \\
\cline { 2 - 2 } & Annotate Wiki Pages as whole \\
\cline { 2 - 3 } & Annotate Wiki pages as parts \\
\hline
\end{tabular}

of TiddlyWiki system. Both systems will continue to work autonomously, but new services will emerge from both of them like the ability to index, share, and annotate Wiki Pages.

\section{System of Information Systems versus Service Oriented Architecture}

\subsection{Definition of Service Oriented Architecture}

The Organization for the Advancement of Structured Information Standards (OASIS) [cov, 2015] defines Service Oriented Architecture (SOA) as the following:

"A paradigm for organizing and utilizing distributed capabilities that may be under the control of different ownership domains. It provides a uniform means to offer, discover, interact with and use capabilities to produce desired effects consistent with measurable preconditions and expectations."

The support of automated business integration by web services developments and standards [Mockford, 2004] [Burbeck, 2000] has driven major technological advances in the integration software space, most notably, the service-oriented architecture (SOA). SOA is designed to allow developers to overcome many distributed enterprise computing challenges including application integration, transaction management, security policies, while allowing multiple platforms and protocols and leveraging numerous access devices and legacy systems [Erl, 2004]. The driving goal of SOA is to eliminate these barriers so that applications integrate and run seamlessly.

The most important aspect of SOA is that it separates service implementation from its interface. Service consumers view a service as an endpoint that respond to a particular request. They are not concerned with how the service goes about executing their requests; they only expect the result [Valipour et al., 2009]. 
We aim to explore the similarities, differences, and limitation of SoIS and SOA in terms of the following characteristics [Valipour et al., 2009] [Papazoglou and Van Den Heuvel, 2007] [Jian et al., 2010]:

- Service Discoverability: A service consumer that needs a service discovers what service to use based on a set of criteria at runtime.

- Modularity: A service supports a set of interfaces. These interfaces should be cohesive, meaning that they should all relate to each other in the context of a module.

- Loose Coupling: Coupling refers to the number of dependencies between modules.

- Interoperability: The ability to communicate information between different services/systems regardless of the format or presentation of information.

- Location Transparency: Consumers of a service do not need to know the location of the service when they invoke the service.

- Supporting Environment: The technical environment in which services/systems operate.

- Autonomy: The services/systems work independently without interference from outside factors.

Table 3. Comparsion between SoIS and SOA.

\begin{tabular}{|l|l|l|}
\hline \multicolumn{1}{|c|}{ Characteristics } & \multicolumn{1}{c|}{ SoIS } & \multicolumn{1}{c|}{ SOA } \\
\hline Service Discoverability & $\begin{array}{l}\text { Fully Supported } \\
\text { (Systems and Services level) }\end{array}$ & $\begin{array}{l}\text { Fully Supported } \\
\text { (Services level only) }\end{array}$ \\
\hline Modularity & Not Supported & Fully Supported \\
\hline Loose Coupling & Fully Supported & Fully Supported \\
\hline Interoperability & Fully Supported & Fully Supported \\
\hline Location Transparency & Fully Supported & Fully Supported \\
\hline Supporting Environment & Fully Supported & Fully Supported \\
\hline Autonomy & $\begin{array}{l}\text { Fully Supported } \\
\text { (Systems and Services level) }\end{array}$ & $\begin{array}{l}\text { Fully Supported } \\
\text { (Services level only) }\end{array}$ \\
\hline
\end{tabular}

\subsection{Similarities}

The core strengths of both SoIS and SOA lie in their ability to enhance proper integration, while promoting flexibility. Both of them share similar characteristics, while maintaining different strategies to reach their goals.

In terms of service discoverability, SOA supports the concept of service discovery by providing a registry of services accessible by the service consumer. In SoIS, this registry should be present with the addition to register the whole system and not only its services [Valipour et al., 2009]. 
Modularity is a key concept in SOA. It is an important concept to take into consideration when creating services. Since the concept of modularity is related to the creation of services in the first place, it is hard to incorporate it with SoIS as the architecture of SoIS is concerned with aggregating services already exist in their respected systems [Jamshidi, 2011].

Both SoIS and SOA provide support for the characteristics of Loose Coupling, Interoperability Location Transparency, and Supporting Environment. All of these characteristics should be found in systems produced by SoIS or SOA [Jamuna and Ashok, 2009].

When it comes to autonomy, SoIS emphasizes on the autonomy of encompassing Information Systems as a whole. In SOA autonomy is enforced on services level [Erl, 2008].

\subsection{Differences}

The differences between SoIS and SOA lie in their consideration for the building blocks for the system they aim to construct [Erl, 2008]. While SOA consider services to be dependent building blocks that collaborate to deliver functionality, SoIS focuses on Information Systems as black boxes and use them as building blocks. It is also important to note that SOA provides a framework for system development. It is important to plan for interoperability challenges at an early stage. However, for SoIS the systems already exist and the interoperability problems are only addressed at later stages. Therefore, due to overhead restriction on architecture and a required common communication medium, it is often that individual software models are created for the various systems comprising the SoIS.

\subsection{Limitations}

SoIS engineering faces significant challenges. On one hand, the lack of standards that guide the construction of a complex system constituted of operational independent Information Systems. On the other hand, there is also a lack of standards in regards for evaluation methods for the architecture and performance of the SoIS. However, there is a potential in using SOA as a well-established framework to compensate the lack of standards present currently in the domain of SoIS.

\section{Discussion}

In the previous section we presented the SoIS with an example that will grant users access to various Information Systems from a single interface. The question here is: How can we evaluate our architecture and the measure the performance of the overall system? Performance optimization of the SoIS architecture in order to achieve a common goal or mission has become the focus of various application 
areas. In the optimization problem, the most basic work is quantitative objective evaluation of the alternatives [Jian et al., 2010]. For SoIS, objective evaluation confronts several challenges comparing with single system objective evaluation. These challenges are listed as follows:

- SoIS is aiming at future common goal. It consists of heterogeneous component systems which can be divided into as-is and to-be systems. So the components and boundary of SoIS is evolving with time going.

- There exist ad-hoc interconnections and interactions of multiple integrated complex systems as part of the whole SoIS. So its very difficult to find an analytic formulation to measure the objective value of SoIS alternative.

For SOA complex systems there have been established methods for the definition of metrics that can be utilized to evaluate the architecture and performance of the system. It is possible to deploy those methods in order to evaluate the work done under SoIS architecture.

\section{Conclusion and Future Work}

Our goal was to take current Information Systems and move towards System of Information Systems (SoIS) to aggregate services and exchange information with simplicity and ease. The aim was focused towards providing an architectural model of SoIS to guide such migration. To achieve this goal we undertake this research to determine what is currently known about SoIS and deploy this knowledge to present an example of a SoIS composed of various Information Systems. We found potential to the SoIS to expand and hold new Information Systems. It was also clear that combining services from various Information Systems will result in an added value to users not present when those systems were operating separately.

The next step is to expand our work and introduce new Information Systems to the SoIS based on the example presented in this study and users needs. It should also be tested in a real world experiment. The SoIS should keep simple interface, with all the services as far from the user as a single click, to keep the users experience useful and friendly. Furthermore, we need to apply evaluation metrics used in SOA in the field of SoIS to compensate the absence of such metrics in the newly emerged concept of SoIS.

\section{References}

[cov, 2015] (2015). Organization for the advancement of structured information standards. https://www .oasis-open.org/.

[tid, 2015] (2015). Tiddlywiki non-linear personal web notebook. http://tiddlywiki. $\mathrm{com} /$.

[Ala Atrash, 2014] Ala Atrash, Marie-Hélène Abel, C. M. (2014). Supporting organizational learning with collaborative annotation. In 6th international conference on knowledge management and information sharing (KMIS), pages 237- 244. 
[Bagnoli et al., 2006] Bagnoli, F., Jipsen, P., and Sterbini, A. (2006). Tiddlywiki in science education.

[Bowen and Sahin, 2010] Bowen, R. M. and Sahin, F. (2010). A net-centric xml based system of systems architecture for human tracking. In System of Systems Engineering (SoSE), 2010 5th International Conference on, pages 1-6. IEEE.

[Breschi and Malerba, 1996] Breschi, S. and Malerba, F. (1996). Sectoral innovation systems: technological regimes, Schumpeterian dynamics and spatial boundaries. Centro studi sui processi di internazionalizzazione, Università commerciale'Luigi Bocconi'.

[Burbeck, 2000] Burbeck, S. (2000). The tao of e-business services: The evolution of web applications into service-oriented components with web services. Online document, IBM Software Group.

[Carlock and Fenton, 2001] Carlock, P. G. and Fenton, R. E. (2001). System of systems (sos) enterprise systems engineering for information-intensive organizations. Systems engineering, 4(4):242-261.

[Carlsson and Stankiewicz, 1991] Carlsson, B. and Stankiewicz, R. (1991). On the nature, function and composition of technological systems. Journal of evolutionary economics, 1(2):93-118.

[Erl, 2004] Erl, T. (2004). Service-oriented architecture: a field guide to integrating $X M L$ and web services. Prentice Hall PTR.

[Erl, 2008] Erl, T. (2008). Soa: principles of service design, volume 1. Prentice Hall Upper Saddle River.

[Jamshidi, 2011] Jamshidi, M. (2011). System of systems engineering: innovations for the twenty-first century, volume 58. John Wiley \& Sons.

[Jamuna and Ashok, 2009] Jamuna, R. S. and Ashok, M. S. (2009). A survey on service-oriented architecture for e-learning system. In Intelligent Agent $\&$ MultiAgent Systems, 2009. IAMA 2009. International Conference on, pages 1-3. IEEE.

[Jian et al., 2010] Jian, X., Bing-feng, G., Xiao-ke, Z., Ke-wei, Y., and Ying-Wu, C. (2010). Evaluation method of system-of-systems architecture using knowledge-based executable model. In Management Science and Engineering (ICMSE), 2010 International Conference on, pages 141-147. IEEE.

[Manthorpe, 1996] Manthorpe, W. H. (1996). The emerging joint system of systems: A systems engineering challenge and opportunity for apl. Johns Hopkins APL Technical Digest, 17(3):305.

[Mishra and Alok, 2011] Mishra, D. and Alok, M. (2011). Research trends in management issues of global software development: evaluating the past to envision the future. Journal of Global Information Technology Management, 14(4):48-69.

[Mishra and Mishra, 2009] Mishra, D. and Mishra, A. (2009). Distributed information system development: review of some management issues. In On the Move to Meaningful Internet Systems: OTM 2009 Workshops, pages 282-291. Springer.

[Mockford, 2004] Mockford, K. (2004). Web services architecture. BT Technology Journal, 22(1):19-26.

[Papazoglou and Van Den Heuvel, 2007] Papazoglou, M. P. and Van Den Heuvel, W.J. (2007). Service oriented architectures: approaches, technologies and research issues. The VLDB journal, 16(3):389-415.

[Rechtin and Maier, 2000] Rechtin, E. and Maier, M. W. (2000). The art of systems architecting. CRC Press.

[Valipour et al., 2009] Valipour, M. H., AmirZafari, B., Maleki, K. N., and Daneshpour, N. (2009). A brief survey of software architecture concepts and service oriented architecture. In Computer Science and Information Technology, 2009. ICCSIT 2009. 2nd IEEE International Conference on, pages 34-38. IEEE 